\section{Proformas negativas e interrogativas em Sateré-mawé*}

\author{
Negative and interrogative \\ proforms in Sateré-mawé
}

Denize de Souza CARNEIRO (UFOPA) dchiaretta@hotmail.com

Fernanda Ferreira SPOLADORE (UFU) fernandafspoladore@hotmail.com
CARNEIRO, Denize de Souza;

SPOLADORE, Fernanda Ferreira.

Proformas negativas e interrogativas

em Sateré-mawé. Entrepalavras,

Fortaleza, v. 7, p. 36-53, ago./dez.

Resumo: Este artigo tem como objetivo apresentar a sistematização de uma análise da correlação morfossemântica das proformas interrogativas e negativas da língua SateréMawé, cuja população corresponde a aproximadamente doze mil pessoas. Os sateré-mawé vivem na Terra Indígena Andirá-Marau, situada na divisa do Amazonas com o Pará, e sua língua é classificada como membro único da família Mawé, integrante do tronco linguístico Tupi. A referida análise foi feita com base em um corpus constituído de textos orais e escritos. Os textos orais foram coletados em contextos reais de comunicação; e os escritos, produzidos por professores que atuam nas comunidades da área indígena. A correlação existente entre a interrogação e a negação no sistema linguístico do SateréMawé é bastante interessante, tendo em vista que as proformas negativas são formadas a partir das proformas interrogativas por meio da afixação do morfema it.... i, indicador de negação. Notavelmente, as proformas negativas não mantêm apenas as características morfológicas das proformas interrogativas, mas também o valor semântico básico delas, conforme mostraremos neste texto.

Palavras-chave: Negação. Interrogação. Língua Sateré-Mawé.

* Fruto da comunicação Proformas interrogativas e negativas em Sateré-Mawé apresentada no XXIX Encontro Nacional da ANPOLL, realizado nos dias 09, 10 e 11 de julho de 2014 em Florianópolis/SC. 
Abstract: The aim of this article is to present the systematization of an analysis of the morphosemantic correlation of interrogative and negative proforms of Sateré-Mawé language, whose population corresponds to approximately 12 thousand people. SateréMawé people live in Andirá-Marau Indigenous Reserve, located in the border region of the Amazonas and Para states, and its language is classified as the only member of Mawé family, constituent of linguistic trunk Tupi. The referred analysis was based in a corpus, constituted by oral and written texts. The oral texts were collected in real contexts of communication and the written ones were produced by teachers who act in the communities from indigenous area. The correlation between interrogation and negation in Sateré-Mawé linguistic system is considerable interesting, since negative proforms are formed from interrogative proforms through the affixation of the morpheme it...Pi, negation indicator. Notably, the negative proforms don't just keep the morphologic characteristics of the interrogative proforms, but also their basic semantic value, as we will present in this text.

Keywords: Negation. Interrogation. Sateré-Mawé language.

\section{Introdução}

As proformas interrogativas e negativas nas línguas indígenas brasileiras, inclusive nas línguas do tronco Tupi (que apresentam mais estudos), ainda não se encontram suficientemente descritas. Quando realizados, os estudos acerca da interrogação e negação aparecem, em geral, em subtópicos de dissertações e teses cujo foco é a descrição e análise morfossintática de uma língua como um todo. Uma vez que a compreensão desses subsistemas é geralmente secundária, os trabalhos, em sua maioria, não apresentam uma descrição detalhada da interrogação e da negação, portanto, falta-lhes a sistematização de um conhecimento mais aprofundado a respeito das proformas interrogativas e negativas.

Dito isso, este artigo apresenta uma descrição e análise das proformas negativas e interrogativas da língua Sateré-Mawé, com a finalidade de mostrar que as proformas negativas ${ }^{1}$ são construídas morfologicamente a partir de proformas interrogativas², adicionandose a estas o morfema indicador de negação it...pi. É interessante destacar que as proformas negativas não preservam apenas a forma morfológica das proformas interrogativas, mas também mantêm o valor semântico básico delas.

\footnotetext{
${ }^{1}$ As proformas negativas são palavras que trazem em si mesmas uma natureza negativa (CREISSELS, 2006), como ocorre em Português com os seguintes termos: nenhum, ninguém, nunca, nada.

2 As proformas interrogativas são empregadas para questionar informação que preencha a lacuna de um enunciado (CREISSELS, 2006), assim como as palavras do Português o que, quem, onde, quando, entre outras.
} 
v. $7(2)$

36-53

ago/dez

2017

A compreensão do sistema negativo do Sateré-Mawé é fruto das pesquisas de Carneiro (2012), Carneiro \& Franceschini (2015) e Carneiro \& Franceschini (2016), ao passo que as pesquisas de Spoladore (2011) e Franceschini \& Spoladore (2016) contribuíram para o entendimento do sistema interrogativo dessa língua. Tais pesquisas foram realizadas por meio de um corpus constituído de textos orais, coletados em situações reais de comunicação, e escritos, autoria de professores e lideranças indígenas deste grupo.

A língua Sateré-Mawé foi classificada por Aryon Dall'Igna Rodrigues (2002) como o único membro da família linguística Mawé, pertencente ao tronco Tupi. Falada pelo povo Sateré-Mawé, é uma das sobreviventes dentre as mais de mil línguas indígenas faladas no início da colonização portuguesa. De acordo com dados da Secretaria Especial de Saúde Indígena (SESAI/Parintins, 2014)3 , a população sateré-mawé equivale a cerca de doze mil pessoas, as quais habitam a região do médio rio Amazonas, na Terra Indígena Andirá-Maraú4, situada na divisa dos estados do Amazonas e Pará.

Com o intuito de avançar na documentação da língua SateréMawé, este artigo traz uma proposta de descrição e análise de suas proformas, ilustrando que as negativas são constituídas a partir das interrogativas. Assim, a fim de mostrar essa estratégia linguística, apresentamos brevemente a sistematização da interrogação e da negação para, em seguida, apresentar a relação morfossemântica de suas proformas.

\section{A interrogação nas línguas}

Segundo Payne (1997, p. 299), são denominados interrogativos os enunciados que estabelecem qualquer modo de questionamento, cabendo-lhes as seguintes funções: (a) solicitação de ação específica; (b) o pedido/confirmação de determinada informação; (c) bem como a obtenção de efeito retórico.

De acordo com Creissels (2006, p. 170), em línguas descritas e analisadas, os enunciados interrogativos são basicamente de dois tipos: totais e parciais. As perguntas totais, aquelas respondidas por sim ou não, podem ser assinaladas por diferentes recursos fonológicos

\footnotetext{
3 Informação oral, obtida na Sede da SESAI de Parintins/AM.

4 Homologada em 06 de agosto de 1986, sua demarcação compreende 788.528 hectares (ha) e 477,7 km de perímetro (TEIXEIRA, 2005).
} 
e/ou morfossintáticos, a seguir: entonação especial, acréscimo de partícula interrogativa, ordenamento de palavras, entre outros (PAYNE, 1997, p. 295).

As perguntas parciais, por sua vez, são caracterizadas por uma lacuna de conteúdo, devendo ser preenchidas com a informação que lhes falta (CREISSELS, 2006, p. 170). São reconhecidas basicamente pelo emprego de palavra ${ }^{5}$ interrogativa, cuja função é interrogar a respeito da informação não compartilhada entre locutor e interlocutor. Em certas línguas, palavra e partículas interrogativas podem coocorrer no mesmo enunciado (GIVÓN, 2001, p. 296).

A seguir, apresentamos brevemente as perguntas totais do Sateré-Mawé, assinaladas ${ }^{6}$ pelas partículas interrogativas in ou apo. Em seguida e, principalmente, ilustramos as perguntas parciais empregadas nessa língua, caracterizadas pelo emprego de diferentes proformas interrogativas, acompanhadas ou não de partícula dessa natureza.

\section{A interrogação em Sateré-Mawé7}

Em Sateré-Mawé, os enunciados interrogativos podem ser de dois tipos: (a) total, tipicamente respondido com sim ou não; e (b) parcial, cuja resposta do interlocutor deve preencher a lacuna deixada pelo locutor.

Os enunciados interrogativos podem ser assinalados pelas partículas in ou apo, cuja função, além de indicar que o enunciado é interrogativo, consiste em marcar o escopo da interrogação. Distinguemse, entretanto, pela compatibilidade da partícula in com a partícula enunciativa $\boldsymbol{k e}^{8}$, incompatível com a partícula apo.

Os enunciados a seguir são interrogativo-total e parcial, respectivamente.

\footnotetext{
${ }^{5}$ Neste artigo, proforma interrogativa.

${ }^{6}$ Não tratamos neste artigo a respeito da entonação empregada nos enunciados sateré-awé.

7 Tópico organizado com base nos estudos de Spoladore (2011) e Franceschini \& Spoladore (2016). Os enunciados interrogativos, em sua maioria, foram retirados dos seguintes livros monolíngues em Sateré-Mawé: Satere-Mawe mowe'eg hap; Wantym sa'awy etiat; Warana sa'awy etiat; Satere-Mawe pusu ag̃kukag̃; Wahemeikowo tuerut aheko e Satere Miwan Pakup. Outros dados apresentados foram coletados em pesquisas de campo, em situações naturais de comunicação.

8 Ao empregar a partícula enunciativa ke, o locutor suspeita da resposta de seu interlocutor, "esperando receber uma confirmação em relação as suas expectativas" (SPOLADORE, 2011, p. 127).
} 


\begin{tabular}{c|c|c|c|c|l|l|l|l}
$(1)$ & $\mathbf{e}$ & - & $\mathbf{t u}$ & - & 'u & pira & apo \\
\hline & 2Ag. & + & At.T & + & 'comer' & 'peixe' & Inter. \\
\hline & "Você comeu o peixe?" \\
\hline & & & & & & & & \\
\hline$(2)$ & $\mathbf{i}$ & - & $\mathbf{i}$ & - & kahu & ke & in & wahi \\
\hline & 3Inat. & + & Atr.II & + & 'ser bonito' & Enunc. & Inter. 'colar' \\
\hline & "É bonito o colar?"
\end{tabular}

Os enunciados em (1) e (2) são interrogativo-totais. Em (1), o verbo ativo $\boldsymbol{e t u}^{\prime} \boldsymbol{u}$ seleciona dois actantes ${ }^{9}$ : o morfema de segunda pessoa do singular $\{\boldsymbol{e}-\}$, índice actancial no papel de agente; e o sintagma nominal pira 'peixe, segundo actante no papel de paciente. São seguidos pela partícula interrogativa apo, cuja incidência é sobre todo o enunciado. Em (2), por sua vez, emprega-se o verbo ikahu. Estativo, seleciona o sintagma nominal wahi 'colar', actante único no papel de qualificado e em concordância com o morfema de terceira pessoa do singular $\{\boldsymbol{i}-\}$. A partícula interrogativa in é empregada após o sintagma verbal $\mathbf{i k a h u}$, mas em posição anterior à do sintagma nominal wahi, o que indica que a interrogação incide sobre o verbo. Além disso, o emprego da proforma enunciativa $\boldsymbol{k} \boldsymbol{e}$ indica que o locutor, ao realizar a pergunta em questão, suspeita da resposta de seu interlocutor.

\begin{tabular}{l|c|c|c|c|c|c|c|c|c|c|c|c|c} 
(3) & karania & in & pira & $\emptyset$ & - & he & - & miariru & $\boldsymbol{\emptyset}$ & - & $\mathbf{t}$ & - & eru:t \\
\hline & 'quantos' & Inter. & 'peixe' & 3poss. & + & Atr.I & + & 'neto' & 3Ag. & + & At.T & + & 'trazer' \\
\hline
\end{tabular}

Em (3), interrogativo-parcial, o verbo ativo teru:t seleciona os sintagmas nominais hemiariru 'neto dele', primeiro actante no papel de agente, e pira 'peixe', segundo actante no papel de paciente. A proforma karania 'quantos', por sua vez, substitui o determinante do nominal pira sobre o qual interroga, uma vez que, semanticamente, questiona sobre a quantidade de peixes. A partícula in, em enunciados interrogativoparciais, ocorre na posição seguinte à da proforma interrogativa; nesse enunciado, acompanha a proforma karania.

Em Sateré-Mawé, os enunciados interrogativo-parciais são caracterizados pelo emprego de diferentes proformas interrogativas, especificadas no quadro $\mathrm{A}$, a seguir. 
Quadro A - As proformas interrogativas do Sateré-Mawé.

\begin{tabular}{|c|c|c|}
\hline Nível morfológico & \multicolumn{2}{|c|}{ Nível semântico-referencial } \\
\hline kat $\sim$ kan & que, o que & referente não humano \\
\hline uwe & quem & referente humano \\
\hline aikope & onde & \multirow{2}{*}{ localização espacial } \\
\cline { 1 - 1 } asuwe & para onde & \\
\hline ainupe & por onde & \\
\hline aikowo & de onde & \\
\hline aikopuo & como & Modo \\
\hline aimepuo & aikope pii & quantidade \\
\hline aikotã & quando (as), (os) & localização temporal \\
\hline karania & por que & Causa \\
\hline karãpe & & \\
\hline kat pote & &
\end{tabular}

Fonte: Spoladore (2012, p. 129).

Para o que se pretende neste artigo - mostrar a relação entre as proformas negativas e interrogativas da língua Sateré-Mawé -, trataremos apenas das proformas interrogativas ${ }^{10}$ kat, uwe, aikotã e karãpe, apresentadas separadamente a seguir.

A proforma interrogativa kat

Emprega-se a proforma kat 'que, o que' para se questionar acerca de um referente não humano, como exemplificado a seguir.

\begin{tabular}{l|l|l|l|l|l|l|c|c|c|c|c|c}
$(4)$ & kat & ke & in & mankuka & $\boldsymbol{\emptyset}$ & - & ti & - & tek & - & tek & \\
\hline & 'o que' & Enunc. & Inter. & 'raposa' & $3 \mathrm{Ag}$. & + & At.T & + & 'cortar' & + & dupl. & \\
\hline
\end{tabular}

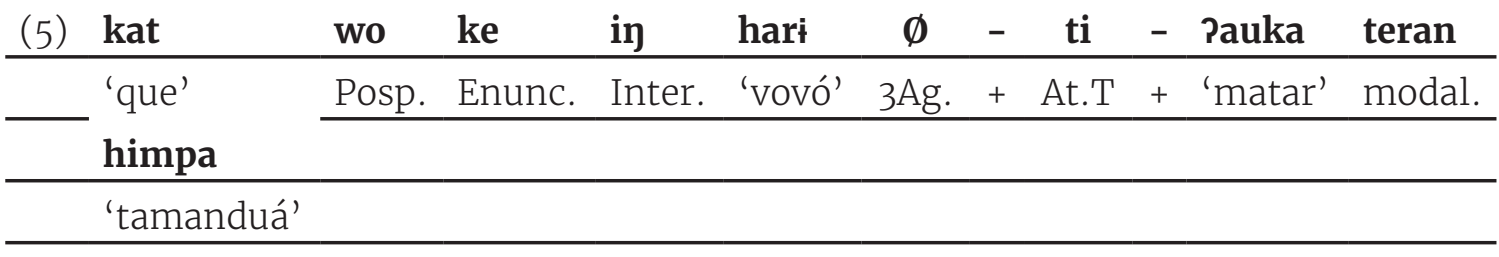

"Com o quê a vovó queria matar o tamanduá?"

Ambos os enunciados supracitados empregam a proforma kat 'que, o que'. Em (4), substitui o sintagma nominal, em função

${ }^{10}$ A respeito das outras proformas da língua Sateré-Mawé, ver Spoladore (2011) e Franceschini \& Spoladore (2016). 
v. 7 (2)

36-53

ago/dez 2017

actancial, sobre o qual interroga. Como ativo, o verbo titektek seleciona dois actantes: o sintagma nominal maykuka 'raposa', empregado no papel de agente, e a proforma kat, em substituição ao actante no papel de paciente. Por sua vez, o enunciado em (5) emprega o sintagma posposicionado kat wo, em função circunstancial, que interroga a respeito do instrumento a ser utilizado pela vovó para matar o tamanduá. O verbo tipauka, ativo, seleciona os sintagmas nominais hari 'vovó', primeiro actante no papel de agente, e himpa 'tamanduá', segundo actante no papel de paciente. Em ambos os enunciados, a proforma kat é seguida das partículas ke, enunciativa, e in, interrogativa.

A proforma interrogativa uwe

A proforma uwe 'quem' é empregada para se interrogar acerca de um referente humano. Os enunciados abaixo são caracterizados pelo emprego desta proforma.

\begin{tabular}{c|c|c|c|c|c|c|c|l|l} 
(6) & uwe & in & $\emptyset$ & - & $\mathbf{i}$ & - & koi & teren-te?en & mani \\
\hline & 'quem' & Inter. & 3Inat. & + & Inv. & + & 'plantar' & Asp. & 'mandioca' \\
\hline \multicolumn{10}{l}{ "Quem sempre planta mandioca?" }
\end{tabular}

\begin{tabular}{c|c|c|c|l|l|c|c|c} 
(7) & uwe & ke & in & tapatu & - & $\emptyset$ & - & suk \\
\hline & 'quem' & Enunc. & Inter. & 3pl.cor.Ag. & + & At.T & + & 'furar' \\
\hline & "Quem foi que eles furaram?"
\end{tabular}

Os enunciados acima são caracterizados pelo emprego da proforma uwe 'quem'. Em (6), o verbo ativo ikoi seleciona dois actantes: a proforma uwe, que substitui e interroga o actante no papel de agente; e o sintagma nominal mani 'mandioca', empregado no papel de paciente. O enunciado em (7), por seu turno, emprega o verbo tapatusuk. Como verbo ativo, seleciona o morfema de terceira pessoa do plural \{ta'atu-\}, índice actancial no papel de agente, e a proforma uwe, que substitui e interroga o actante no papel de paciente. Em (6), a proforma uwe é acompanhada da partícula interrogativa in; em (7), por sua vez, a partícula in é antecedida da partícula ke, enunciativa.

A proforma interrogativa aikotã

Emprega-se a proforma interrogativa aikotã 'como' para se interrogar a respeito da circunstância modal: (a) de ser/estar de um 
referente, humano ou não humano; ou (b) de se realizar um evento. Abaixo, os enunciados ilustram o emprego dessa proforma:

\begin{tabular}{r|c|c|c|c|c|c|c|c} 
(8) & aikotã & ke & in & hari & cristina & e & - & ko \\
\hline & 'Como' & Enunc. & Inter. & 'vovó' & 'Cristina' & Atr.I & + & 'história' \\
\hline \multicolumn{6}{l}{ "Como [é] a história da vovó Cristina?" }
\end{tabular}

\begin{tabular}{r|l|l|l|l|c|l|l|l} 
(9) & aikotã & ase?i & $\emptyset$ & - & ti & - & Pauka & himpa \\
\hline & 'como' & 'vovô' & 3Ag. & + & At.T & + & 'matar' & 'tamanduá' \\
\hline \multicolumn{6}{l}{ "Como o vovô matou o tamanduá?" }
\end{tabular}

Ambos os enunciados supracitados empregam a proforma aikotã 'como'. Em (8), enunciado não verbal, a proforma aikotã substitui o sintagma, em função predicativa, sobre o qual interroga (o modo de ser da história da vovó Cristina). É seguido do sintagma nominal hari cristina eko 'a história da vovó Cristina', empregado em função actancial. Em (9), a proforma aikotã substitui o sintagma, em função circunstancial, sobre o qual interroga (o modo de se realizar a ação de matar o tamanduá). Nesse enunciado, o verbo ativo tipauka é responsável por selecionar dois actantes: o sintagma nominal ase pi 'vovô', empregado no papel de agente e em concordância com o morfema de terceira pessoa $\{\emptyset-\}$; e o sintagma nominal himpa 'tamanduá', empregado no papel de paciente.

A proforma interrogativa karãpe

O emprego da proforma karãpe 'quando' é condicionado pela intenção do locutor em questionar a respeito da circunstância temporal da realização de um evento. Os enunciados a seguir são caracterizados pelo emprego desta proforma:

\begin{tabular}{r|c|c|c|c|c|c|l|l} 
(10) & karãpe & wawori & $\emptyset$ & - & ti & - & puenti & wewato \\
\hline & 'quando' & 'jabuti' & 3Ag. & + & At.T & + & 'encontrar' & 'anta' \\
\hline \multicolumn{6}{l}{ "Quando o jabuti encontrou a anta?" }
\end{tabular}

\begin{tabular}{r|c|c|c|c|c|c|c|c|c|c|c} 
(11) & karãpe & ke & in & hari & $\emptyset$ & - & to & - & to & miat & pe \\
\hline & 'quando' & Enunc. & Inter. & 'vovó' & $3 \mathrm{Ag}$. & + & Med. & + & 'ir' & 'caça' & Posp. \\
\hline & "Quando a vovó foi para a caça?"
\end{tabular}


v. $7(2)$

36-53

ago/dez

2017

Acima, a proforma karãpe 'quando', empregada em função circunstancial, interroga acerca da localização temporal dos eventos encontrar a anta e ir para a caça, respectivamente. Em (10), o verbo ativo tipuenti seleciona dois sintagmas nominais: wawori 'jabuti', primeiro actante no papel de agente, e wewato 'anta', segundo actante no papel de paciente. O enunciado em (11), por sua vez, emprega o verbo médio toto, cujo sentido é preenchido pelo sintagma nominal hari 'vovó', actante no papel de agente/paciente e em concordância com o morfema de terceira pessoa $\{\emptyset$ - $\}$. Em posição final, o sintagma posposicionado miat pe 'para a caça' funciona como circunstancial locativo.

\section{A negação nas línguas}

A expressão da negação nas línguas pode ser feita por diferentes mecanismos linguísticos, sejam estes gramaticais ou lexicais.

Gramaticalmente, a negação pode ocorrer no nível da formação de palavras ou da sintaxe. No primeiro, a negação ocorre em línguas que apresentam processo sistemático de derivação de antônimos com sentido negativo. No entanto, há línguas que não possuem esse mecanismo; é o caso das muitas línguas africanas subsaarianas, nas quais a expressão da negação ocorre apenas por meio de mecanismos morfossintáticos. Porém, todas as línguas parecem possuir em seu léxico pares de antônimos, geralmente de verbos (como, por exemplo, continuar/cessar; aceitar/recusar) com sentido negativo. Entre os valores de verdade de duas frases construídas por um par de predicados antônimos há a mesma relação que há entre uma frase positiva e sua contrapartida negativa (CREISSELS, 2006, p. 131).

Lexicalmente, segundo Payne (1997, p. 282), a negação descreve uma situação em que o conceito de negação faz parte da semântica lexical de um termo do léxico, particularmente do verbo, como, por exemplo, o verbo faltar, ocorrendo como negação do verbo ter. Creissels (2006), ao contrário de Payne, considera que a negação de frase - todo enunciado que apresentar uma negação não restrita ao grupo verbal, podendo incidir sobre qualquer elemento do enunciado - é a única necessária ao funcionamento da negação em uma língua, e, portanto, a análise desse tipo de negação é que permitirá chegar aos diferentes mecanismos morfossintáticos, isto é, às diversas formas linguísticas que têm a função de negar em uma determinada língua.

De acordo com esse autor, tais mecanismos aparecem nas línguas 
pela supressão de formas, que se manifestam na asserção afirmativa e que são suprimidas na asserção negativa, ou pelo acréscimo de formas de negação às asserções afirmativas correspondentes, podendo, nesse caso, a negação ser realizada: (1) por formas dependentes / partículas; (2) por formas presas (negação morfológica); (3) por auxiliares de negação (CREISSELS, 2006).

O estudo das construções negativas em Sateré-Mawé mostrou que a negação nessa língua é expressa por meio do acréscimo do morfema it...Pi, incluindo-a no grupo das línguas que expressam a negação por meio do acréscimo de formas às afirmativas correspondentes.

Além desses mecanismos morfossintáticos de expressão da negação, é útil mencionar as proformas negativas, ou seja, os pronomes e os advérbios que apresentam em si mesmos uma natureza negativa conforme as palavras do Português nenhum, ninguém, nunca, nada - e, por essa razão, podem ser usados como resposta negativa a um pedido de afirmação sem, no entanto, necessitar da utilização de qualquer outro indicador de negação (CREISSELS, 2006). É sobre a constituição morfossemântica dessas proformas em Sateré-Mawé que pretendemos abordar a seguir.

A negação em Sateré-Mawén

Em Sateré-Mawé, a negação de enunciados declarativos ${ }^{12}$ é expressa morfologicamente pela inserção do morfema descontínuo it... ii, podendo incidir sobre um sintagma em função actancial, sobre o predicado verbal ou sobre um sintagma em função circunstancial. O morfema it (primeiro segmento) é empregado em posição anterior à do sintagma, enquanto que o morfema pi (segundo segmento) ocorre em posição seguinte à do elemento negado.

Os enunciados do Sateré-Mawé, a seguir, ilustram a negação de enunciados declarativos:

\begin{tabular}{l|c|c|c|c|l|l} 
(12) & ko & - & $\emptyset$ & - & wat & weita \\
\hline & class. & + & afast. & + & rad.gen. & 'pássaro' \\
\hline \multicolumn{6}{l}{ "Este é pássaro." }
\end{tabular}

\footnotetext{
${ }^{11}$ Tópico organizado com base nos estudos de Carneiro (2012), Carneiro \& Franceschini (2015) e Carneiro \& Franceschini (2016).

12 Segundo Carneiro (2012), os enunciados imperativos e optativos do Sateré-Mawé são marcados pelos morfemas descontínuos it...tei?o e it...te, respectivamente.
} 
v. 7 (2)

36-53

ago/dez

2017

\begin{tabular}{l|l|l|l|l|l|l|l} 
(13) & mipu & ran & $\mathbf{i t}$ & - & $\mathbf{m i} \mathbf{i}$ & - & 'i $^{\prime}$ \\
\hline & 'Comida' & 'remosa' & Neg. & + & 'comida' & + & Neg. \\
\hline
\end{tabular}

Os enunciados supracitados são não verbais. Em (12), declarativoafirmativo, são justapostos os sintagmas nominal kowat 'este', em função actancial, e weita 'pássaro', em função predicativa. Em (13), declarativo-negativo, a negação incide sobre o nominal mi?u 'comida', circundado pelo morfema descontínuo-negativo it...pi. Em primeira posição, o sintagma nominal mipu ran 'comida remosa' é empregado em função actancial; em seguida, o sintagma nominal it mi $\mathbf{p u}$ Pi 'não é comida' desempenha função predicativa.

\begin{tabular}{c|c|c|c|c|c|l}
$(14)$ & nilda & $\emptyset$ & - & $\mathbf{i}$ & - & hairu \\
\hline & Nilda & 3Inat. & + & Atr. & + & 'dançar' \\
\hline & "Nilda está dançando."
\end{tabular}

\begin{tabular}{r|c|c|c|c|c|c|c|l|l|l} 
(15) & nilda & $\mathbf{i t}$ & - & $\boldsymbol{\emptyset}$ & - & $\mathbf{i}$ & - & hairu & - & Pi \\
\hline & Nilda & Neg. & + & 3Inat. & + & Atr. & + & 'dançar' & + & Neg. \\
\hline \multicolumn{6}{l}{ "Nilda não está dançando." }
\end{tabular}

Os enunciados acima são verbais, caracterizados pelo emprego de verbo estativo. Em (14), declarativo-afirmativo, emprega-se o sintagma nominal nilda, em função actancial, seguido do sintagma verbal ihairu, em função predicativa. O enunciado em (15), declarativo-negativo, distingue-se do enunciado anterior pelo emprego do sintagma verbal itihairupi, resultado da incidência do morfema descontínuo-negativo it.... Pi sob o verbo ihairu.

\begin{tabular}{c|c|c|c|c|l|l}
$(16)$ & tapatu & - & $\emptyset$ & - & $\mathbf{P u}$ & ariukere \\
\hline & $3 \mathrm{Ag}$. & + & At.T & + & 'comer' & 'preguiça' \\
\hline \multicolumn{6}{|l}{ "Elas comem preguiça." }
\end{tabular}

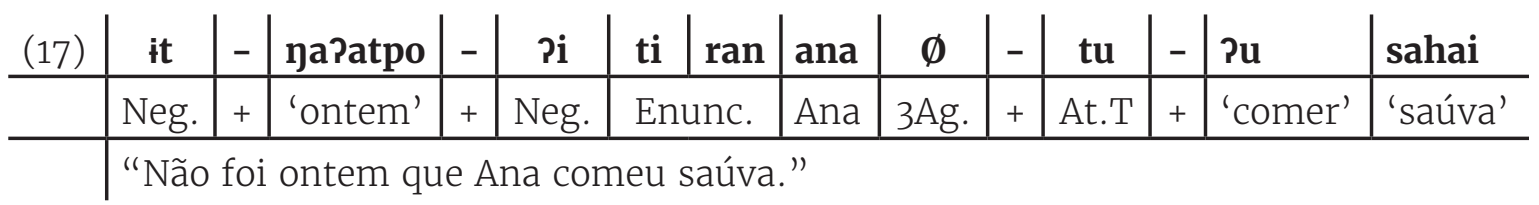

Verbais, os enunciados acima se assemelham pelo emprego de verbo ativo. Em (16), declarativo-afirmativo, o verbo tapatupu seleciona 
dois actantes: o primeiro, expresso pelo morfema de terceira pessoa do plural \{tapatu-\}, no papel de agente; o segundo, expresso pelo sintagma nominal ariukere 'preguiça', no papel de paciente. Em (17), declarativonegativo, o verbo tu $\mathbf{p}$ u seleciona dois sintagmas nominais: ana, primeiro actante no papel de agente, e sahai 'saúva', segundo actante no papel de paciente. Em primeira posição, o morfema descontínuo-negativo it... pi circunda o adverbial napatpo 'ontem', cuja função é circunstancial.

\section{As proformas negativas e interrogativas ${ }^{13}$}

Em Sateré-Mawé, as proformas negativas são construídas a partir de proformas interrogativas, adicionando-se a estas o morfema indicador de negação it...pi. Neste estudo, foram encontradas quatro proformas negativas formadas morfologicamente a partir de proformas interrogativas, cujo valor semântico básico é mantido.

O funcionamento dessas proformas será detalhado adiante.

As proformas kat e itkat?i

Em Sateré-Mawé, emprega-se a proforma interrogativa kat

'que, o que' para se interrogar a respeito de referente(s) não humano(s). Formada a partir da proforma kat e do morfema descontínuo it...Pi, a proforma negativa itkat?i 'nada' é empregada para se negar referente(s) dessa natureza.

O emprego das proformas kat e itkat?i é ilustrado a seguir:

\begin{tabular}{c|c|c|c|c|c|c|l|l|l}
$(18)$ & kat & ke & in & hari & e & - & Paware & - & ria \\
\hline & 'o que' & Enunc. & Inter. & vovó' & Atr.I & + & 'cachorro' & + & plural
\end{tabular}

\begin{tabular}{c|c|c|c|c|c|c|l} 
& $\emptyset$ & - & ti & - & puenti & yapapi & pe \\
\hline & 3pl.Ag. & + & At.T & + & 'encontrar' & 'mato' & Posp. \\
\hline
\end{tabular}

\begin{tabular}{|c|c|c|c|c|c|c|c|c|c|c|c|c|}
\hline (19) & it & - & kat & - & Pi & uru & - & tu & - & Pu & só $^{14}$ & more $^{15}$ \\
\hline & Neg. & + & Prof. & + & Neg. & 1pl.Ag. & + & At.T & + & 'comer' & & 'urupé' \\
\hline
\end{tabular}

\footnotetext{
${ }_{13}$ Tópico organizado com base nos estudos de Spoladore (2011), Carneiro (2012), Carneiro \& Franceschini (2015), Carneiro \& Franceschini (2016) e Franceschini \& Spoladore (2016).

${ }_{14}$ Termo usado no Amazonas para designar um tipo de cogumelo comestível.
} 
v. 7 (2)

36-53

ago/dez

2017

Os enunciados acima são caracterizados pelo emprego das respectivas proformas $\boldsymbol{k} a \boldsymbol{t}$ 'que, o que', interrogativa, e itkat $\boldsymbol{i}$ 'nada', negativa. Interrogativo, o enunciado em (18) emprega a proforma kat, que substitui o sintagma nominal, em função actancial, sobre o qual interroga. Ativo, o verbo tipuenti seleciona dois actantes: o primeiro (no papel de agente), o sintagma nominal hari epawareria 'cachorros da vovó', em concordância com o morfema pessoal $\{\emptyset-\}$, e o segundo (no papel de paciente), substituído/interrogado pela proforma kat. Em posição inicial, é acompanhada das partículas $\boldsymbol{k} \boldsymbol{e}$, enunciativa, e in, interrogativa. Em posição final, o sintagma posposicionado na papipe 'no mato' desempenha função circuntancial locativa. Declarativo-negativo, o enunciado em (19) emprega, em função actancial, a proforma itkat $\mathbf{i}$ 'nada'. Assim como o verbo tipuenti, o verbo ativo urutupu seleciona dois actantes: o primeiro, no papel de agente, expresso pelo morfema de primeira pessoa exclusiva $\{\boldsymbol{u} \boldsymbol{u} \boldsymbol{u}-\}$, e o segundo, no papel de paciente, expresso pela proforma negativa itkat?i.

As proformas uwe e ituwe $\mathrm{i} i$

Em Sateré-Mawé, a proforma interrogativa uwe 'quem' é empregada para se interrogar acerca de referente(s) humano(s). Da mesma forma, a proforma negativa ituwe pi 'ninguém', formada a partir da proforma uwe e do morfema descontínuo it...Pi é empregada para se negar referente(s) dessa natureza.

Os enunciados a seguir ilustram o emprego dessas proformas.

\begin{tabular}{c|c|c|c|c|c|c|l|l|l} 
(20) & uwe & in & $\emptyset$ & - & $\mathbf{i}$ & - & koi & te?en-te?en & mani \\
\hline & 'quem' & Inter. & 3Inat. & + & Inv. & + & 'plantar' & Asp. & 'mandioca' \\
\hline \multicolumn{1}{l}{ "Quem sempre planta mandioca?" }
\end{tabular}

\begin{tabular}{|c|c|c|c|c|c|c|c|c|c|c|c|}
\hline (21) & - & uwe & Pi & rapin & $\emptyset$ & - & $t$ & - ehapat & I kowat & nimo & rapin \\
\hline Neg. & + & Prof. & \begin{tabular}{l|l}
+ & Neg.
\end{tabular} & Asp. & $3 \mathrm{Ag}$. & + & Med. & + 'ver' & 'isso' & pass. & Asp. \\
\hline
\end{tabular}

Os enunciados acima ilustram o emprego das proformas uwe 'quem', interrogativa, e ituwe Pi 'ninguém', negativa. Em (20), enunciado interrogativo, a proforma uwe substitui o sintagma nominal, em função actancial, sobre o qual interroga. O verbo ativo ikoi seleciona dois actantes: a proforma uwe, no papel de agente, e o sintagma 
nominal mani 'mandioca', no papel de paciente. O enunciado ${ }^{15}$ em (21), declarativo-negativo, é formado da proforma ituwe $\boldsymbol{i}$, actante único no papel de experienciador, selecionado pelo verbo médio teha?at.

As proformas aikotã e itaikotã?i

Em Sateré-Mawé, emprega-se as proformas aikotã 'como', interrogativa, e itaikotã Pi 'não assim', negativa, para se interrogar/negar o modo de ser/estar de um referente (humano ou não humano) ou de se realizar um evento. Como se vê, a proforma itaikotã?i é formada a partir da proforma aikotã, acrescida do morfema descontínuo it... Pi.

O emprego das proformas aikotã e itaikotãpi é ilustrado a seguir:

\begin{tabular}{l|c|c|c|l|l|c|l|l} 
(22) & aikotã & ke & in & hari & cristina & e & - & ko \\
\hline & 'como' & Enunc. & Inter. & 'vovó' & 'Cristina' & Atr.I & + & 'história' \\
\hline \multicolumn{7}{|c}{ "Como [é] a história da vovó Cristina?" }
\end{tabular}

\begin{tabular}{r|l|c|c|c|c|c|c|c|c|c|c|} 
(23) & it & - & aikotã & - & pi & tat & aru & e & - & $\boldsymbol{\emptyset}$ & - \\
\hline & Neg. & + & Prof. & + & Neg. & Enunc. & fut. & 2poss. & + & Atr.II & + \\
\hline mempit & mana & i'atu & - & $\boldsymbol{\emptyset}$ & - & ?e & & & & \\
& 'filho' & 'senhora' & 3pl. & + & Med. & + & aux. & & & & \\
\hline
\end{tabular}

Em (22), enunciado interrogativo, a proforma aikotã 'como' substitui o sintagma, em função predicativa, sobre o qual interroga. É seguida das proformas $\boldsymbol{k e}$, enunciativa, e in, interrogativa, e do sintagma nominal hari cristina eko 'a história da vovó Cristina', empregado em função de actante. Em (23), enunciado declarativo-negativo ${ }^{16}$, a proforma itaikotã?i 'não assim' é empregada em função predicativa, seguida da partícula tat, enunciativa, do morfema temporal aru, e do sintagma nominal emempit 'teu filho', em função actancial.

As proformas karãpe e itkarãpe?i

Em Sateré-Mawé, o emprego das proformas karãpe 'quando', interrogativa, e itkarãpe pi 'nunca', negativa, é condicionado pela

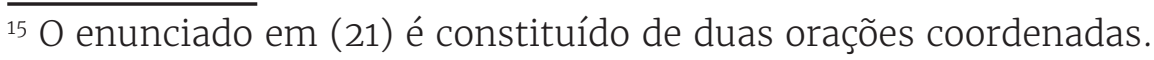

${ }^{16}$ Por meio desse enunciado, "o professor procura consolar a mãe do jovem perfurado, dizendo-lhe que não acontecerá com seu filho o mesmo que aconteceu com outro jovem que veio a falecer por causa de uma perfuração à faca" (CARNEIRO \& FRANCESCHINI, 2016, p. 14). 
v. 7 (2)

36-53

ago/dez 2017

intenção do falante em interrogar ou negar a circunstância temporal da realização de um evento. Veja que a proforma itkarãpe pi é formada a partir da proforma karãpe, acrescida do morfema descontínuo it... Pi, ambas ilustradas nos enunciados a seguir.

\begin{tabular}{l|c|c|c|c|c|c|l|l}
$(24)$ & karãpe & wawori & $\emptyset$ & - & ti & - & puenti & wewato \\
\hline & 'quando' & 'jabuti' & $3 \mathrm{Ag}$. & + & At.T & + & 'encontrar' & 'anta' \\
\hline \multicolumn{6}{|c}{ "Quando o jabuti encontrou a anta?" }
\end{tabular}

\begin{tabular}{l|c|c|c|c|c|c|c|c|c|c|c|c|c|c|} 
(25) & mipi hawii & $\mathbf{i t}$ & - & karãpe & - & pi & rapin & $\boldsymbol{\emptyset}$ & - & to & - & to & miat & pe \\
\hline & 'depois' & Neg. & + & Prof. & + & Neg. & Asp. & 3Ag. & + & Med. & + & (ir & 'caça' & Posp. \\
\hline
\end{tabular}

Em (24), enunciado interrogativo, a proforma karãpe 'quando' substitui o sintagma, em função circunstancial, sobre o qual interroga. O verbo ativo tipuenti seleciona dois actantes, expressos pelos sintagmas nominais wawori 'jabuti', primeiro actante no papel de agente (em concordância com o morfema pessoal $\{\emptyset$ - $\}$ ), e wewato 'anta', segundo actante no papel de paciente. Declarativo-negativo, o enunciado em (25) emprega a proforma itkarãpe pi 'nunca' em função circunstancial temporal. É seguida pelo morfema aspectual rapin e pelo verbo toto, que, sendo médio, seleciona actante único expresso pelo morfema pessoal $\{\emptyset$ - $\}$, índice no papel de agente/paciente. Em posição final, o sintagma posposicionado miat pe 'para a caça' desempenha função circunstancial locativa.

As proformas interrogativas e negativas apresentadas neste artigo estão resumidas no quadro B, a seguir:

Quadro B - As proformas interrogativas e negativas do Sateré-Mawé.

\begin{tabular}{|c|c|}
\hline Proformas Interrogativas & Proformas Negativas \\
\hline $\begin{array}{l}\text { kat 'que, o que' } \\
\text { interroga sobre } \\
\text { referente não humano }\end{array}$ & $\begin{array}{l}\text { it-kat-pi 'nada' } \\
\text { nega } \\
\text { referente não humano }\end{array}$ \\
\hline $\begin{array}{c}\text { uwe 'quem' } \\
\text { interroga sobre } \\
\text { referente humano }\end{array}$ & $\begin{array}{l}\text { it-uwe-pi 'ninguém' } \\
\text { nega } \\
\text { referente não humano }\end{array}$ \\
\hline $\begin{array}{c}\text { aikotã 'como' } \\
\text { interroga sobre } \\
\text { circunstância modal }\end{array}$ & $\begin{array}{c}\text { it-aikotã-pi ' 'não assim' } \\
\text { nega } \\
\text { circunstância modal }\end{array}$ \\
\hline $\begin{array}{c}\text { karãpe 'quando' } \\
\text { interroga sobre } \\
\text { circunstância temporal }\end{array}$ & $\begin{array}{c}\text { it-karãpe-pi 'nunca' } \\
\text { nega } \\
\text { circunstância temporal }\end{array}$ \\
\hline
\end{tabular}

Fonte: Carneiro (2016, p. 6). 


\section{Considerações Finais}

Neste artigo, pretendeu-se apresentar a correlação morfossemântica das proformas interrogativas e negativas do SateréMawé, língua de interação do grupo indígena Mawé, que habita a Terra Indígena Andirá-Marau, localizada na Amazônia brasileira.

A partir da análise de corpus constituído de textos orais e escritos, constatou-sequeas proformas negativas sãoformadas morfologicamente a partir de proformas interrogativas, que, circunfixadas pelo morfema indicador de negação it...pi, tornam-se negativas, mantendo, porém, seu valor semântico básico.

Foram encontradas quatro proformas negativas constituídas por meio desta estratégia, a saber: (1) a proforma it-kat-pi 'nada', empregada para se negar referente(s) não humano(s), é formada a partir da proforma interrogativa kat 'que, o que', que interroga acerca de referente(s) dessa natureza; (2) a proforma $\boldsymbol{i} \mathbf{t}$ - $\boldsymbol{u} \boldsymbol{w} \boldsymbol{e}$ - $\boldsymbol{p i}$ 'ninguém', empregada para se negar referente(s) humano(s), é constituída a partir da proforma interrogativa uwe 'quem', que questiona acerca desse tipo de referente; (3) a proforma it-aikotã-pi 'não assim', empregada para se negar circunstância modal, é formada a partir da proforma interrogativa aikotã 'como', que indaga acerca de circunstância dessa natureza; e (4) a proforma it-karãpe-pi 'nunca', empregada para se negar circunstância temporal, é construída a partir da proforma interrogativa karãpe 'quando', que interroga a respeito desse tipo de circunstância. Nota-se que tanto a forma quanto o sentido das proformas interrogativas são mantidos nas negativas.

\section{Referências}

CARNEIRO, Denise de Souza. A negação em Sateré-Mawé. 2012. Dissertação (Mestrado) - Instituto de Letras e Linguística, Universidade Federal de Uberlândia, Uberlândia, 2012, $119 f$.

CARNEIRO, Denize de Souza; FRANCESCHINI, Dulce do Carmo. Negação e focalização em Sateré-Mawé. Fragmentum (on line), v. 46, p. 38-56, 2015.

CARNEIRO, Denize de Souza; FRANCESCHINI, Dulce do Carmo. Proformas negativas em Sateré-Mawé. Revista do SELL, v. 5, n. 1, 2016.

CREISSELS, Denis. Syntaxe générale une introduction typologique 2. Paris: Lavoisier, 2006.

FRANCESCHINI, Dulce do Carmo; SPOLADORE, Fernanda Ferreira. As proformas interrogativas da língua Sateré-Mawé. Revista do SELL, v. 5, n. 1, 2016. 
V. $7(2)$ 36-53 ago/dez 2017

GIVÓN, Talmy. Non-declarative speech-acts. In: Syntax: an introduction. Amsterdam/Philadelphia: John Benjamins, 2001, v. 2, p. 287326.

LAZARD, Gilbert. L'actance. Paris: Presses Universitaires de France, 1994.

PAYNE, Thomas E. Describing Morphosyntax: A Guide for Field Linguists. Cambridge: Cambridge University Press, 1997, p. 261-305.

RODRIGUES, Aryon D. Línguas brasileiras: Para o conhecimento das Línguas Indígenas. São Paulo: Loyola, 2002.

SPOLADORE, Fernanda Ferreira. A interrogação em Sateré-Mawé. 2011. Dissertação (Mestrado) - Instituto de Letras e Linguística, Universidade Federal de Uberlândia, Uberlândia, 2011, $161 f$.

TEIXEIRA, Pery. (org.). Sateré-Mawé: retrato de um povo indígena. Manaus: UNICEF, 2005.

Recebido em: 15 de fev. de 2017.

Aceito em: 13 de jul. de 2017. 


\section{Anexo}

\begin{tabular}{|c|c|}
\hline \multicolumn{2}{|c|}{ LISTA DE ABREVIATURAS } \\
\hline afast. & Afastamento \\
\hline Ag. & Índice pessoal agentivo \\
\hline Asp. & Aspecto \\
\hline At. & Orientação ativa \\
\hline Atr. & Relação/Orientação atributiva \\
\hline aux. & Verbo auxiliar \\
\hline class. & Classificador \\
\hline cor. & Correferencial \\
\hline dupl. & Duplicação de morfema \\
\hline Enunc. & Partícula Enunciativa \\
\hline fut. & Futuro \\
\hline Inat. & Índice pessoal inativo \\
\hline Inter. & Partícula interrogativa \\
\hline Med. & Orientação média \\
\hline modal. & Modalizador \\
\hline Neg. & Morfema negativo \\
\hline pass. & Passado \\
\hline pl. & Plural \\
\hline plural. & Pluralizador \\
\hline Posp. & Posposição \\
\hline poss. & Morfema possessivo \\
\hline Prof. & Proforma \\
\hline rad. gen. & Radical genérico \\
\hline sg. & Singular \\
\hline
\end{tabular}

